\title{
Self-Sustaining Square-Extensional Mode Resonator Oscillator for Mass Sensing in Liquid ${ }^{\dagger}$
}

\author{
Habiba Begum ${ }^{1, *}$, Abid Ali ${ }^{1}$ and Joshua En-Yuan Lee ${ }^{1,2}$ \\ 1 Department of Electronic Engineering, City University of Hong Kong, Hong Kong 999077, China; \\ abidali2-c@my.cityu.edu.hk (A.A.); josh.lee@cityu.edu.hk (J.E.-Y.L.) \\ 2 State Key Laboratory of Terahertz and Millimeter Waves, City University of Hong Kong, \\ Hong Kong 999077, China \\ * Correspondence: hbegum2-c@my.cityu.edu.hk \\ + Presented at the Eurosensors 2018 Conference, Graz, Austria, 9-12 September 2018.
}

Published: 10 December 2018

\begin{abstract}
We present a 5.41 MHz square-extensional (SE) mode resonator in closed-loop oscillation for resonant mass sensing in the liquid phase. The resonator has been fabricated in piezoelectric thin film aluminum nitride (AIN) on silicon (Si). The strain profile of the SE mode allows for higher electromechanical coupling efficiency using piezoelectric transduction to lower the motional resistance $(R m)$ given the expected low quality factors $(Q)$ in liquid (136 compared to 942 in air). By locking the device into self-sustained oscillation, the minimum detectable frequency shift is reduced from $3680 \mathrm{ppm}$ (open-loop in water) to $8.76 \mathrm{ppm}$ (closed-loop inair).
\end{abstract}

Keywords: MEMS resonator; MEMS oscillator; thin-film piezoelectric-on-silicon (TPoS); liquid phase sensing

\section{Introduction}

The development of biochemical mass sensors has been interest due to their wide scope of applications that cover clinical diagnosis, agriculture \& food quality control, the automotive industry, and contamination detection in polluted water. The sensing environment is a critical consideration, particularly when the sensing must be carried out in a fluidic environment. Microelectromechanical Systems (MEMS) resonators designed to interact with fluids are of interest for sensing the physical properties of liquids [1] and biosensing [2] as they allow for high throughput, real-time monitoring, and require low sample volumes. Ideally, such a resonator should deliver a workable signal-to-noise ratio. As the quality factor $(Q)$ determines the minimum resolvable frequency, it should be as high as possible when the resonator is covered in fluid. Generally, Qs of resonators covered in a fluid are limited to order of 100 at best. This ultimately limits the frequency resolution in the order of $1 \%$ relative to the nominal frequency. Given the abovementioned challenges, various approaches have been explored towards realizing MEMS resonators forhandling fluid samples [3-6].

Pursuant to reducing the minimum resolvable frequency shift, we here demonstrate a MEMS resonant mass sensor implemented in closed-loop by locking the device into self-oscillation. The device under test was square-plate resonator piezoelectrically transduced in the square-extensional (SE) mode [7,8]. By exploiting the enhanced transduction efficiency using piezoelectric transduction, the circuit design is greatly simplified compared to the capacitive resonators described in $[7,8]$, which is particularly critical in the context of applying MEMS resonators as mass sensors in fluids. 


\section{MEMS Resonator Design \& Simulation}

As depicted in Figure 1a, the MEMS sensor comprises a square-plate with a side-length of 800 $\mu \mathrm{m}$ fabricated by MEMSCAP following AlN-on-Si MEMS process. The square-plate resonator comprises a three-layer piezoelectric transducer stack composed of a $10 \mu \mathrm{m}$ thick silicon (Si) device layer, $0.5 \mu \mathrm{m}$ thick aluminum nitride (AlN), and $1 \mu \mathrm{m}$ thick Al. As the Si device layer is surface doped, it acts as the bottom electrode of the MEMS resonator. Using AlN provides the advantage of compatibility with CMOS and stronger potential for chip-level integration compared to other common piezoelectric materials. The Al layer acts as the top electrode. The square plate is supported by T-shaped tethers at each corner to reduce anchor losses to substrate. The resonator is actuated from the input port and the corresponding motional current is picked up through the output port as marked in the micrograph of the MEMS resonator in Figure 1a. The device has been designed to preferentially excite the SE mode, of which the mode shape simulated by COMSOL finite elements (FE) is depicted in Figure 1b. The symmetric lateral strain profile of the SE mode on both the $x$ and $y$ axes enhances the electromechanical coupling efficiency, which lowers the motional resistance $\left(R_{m}\right)$ as desired for implementing an amplifier feedback loop given the reduced values of $Q$ expected in a fluid. The resonant frequency of the SE mode $\left(f_{S E}\right)$ is given by:

$$
f_{S E}=\sqrt{\frac{E}{4 \rho L^{2}}}
$$

where $E$ is the biaxial modulus with reference to the $<110>$ axis in $(100) \mathrm{Si}, \rho$ is the density of $\mathrm{Si}$, and $L$ is the side length of the square plate.

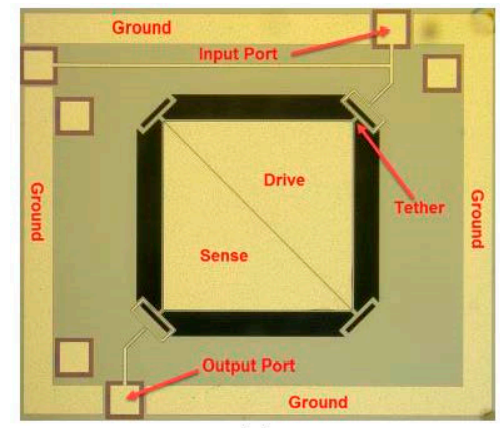

(a)

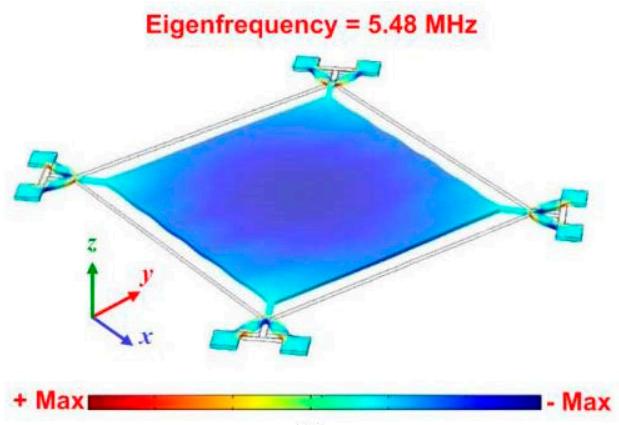

(b)

Figure 1. (a) Micrograph of fabricated square-extensional (SE) mode MEMS resonator; (b) Profile of total sum of lateral strain components $(\varepsilon x+\varepsilon y)$ associated with the SE mode resonance computed by Finite Element (FE) analysis.

\section{Experimental Results}

\subsection{Open-Loop Measurement Result}

The fabricated device was mounted on custom-designed PCB using wire bonds to connect the contact pads and PCB tracks. The electrical transmission characteristics of the resonator in air and water were measured using an Agilent E5061A Network Analyzer using an RF power $0 \mathrm{dBm}$ in both cases. For the measurement in water, the device was fully covered under a droplet of water by pipetting $0.25 \mu \mathrm{L}$ of water. It is worth noting that pipetting beyond $0.15 \mu \mathrm{L}$ of water does not broaden the peak any further. The measured transmission $S_{21}$ magnitude in air and water are depicted in Figure 2, showing a broadening of the peak and reduction in its magnitude when the resonator is covered in water. The measured frequency response curves were fitted to an equivalent circuit model comprising a series resonant circuit $\left(L_{m}, C_{m}\right.$, and $\left.R_{m}\right)$ with two parallel parasitic elements $\left(C_{f}\right.$ and $\left.R_{f}\right)$ to extract the equivalent circuit parameters. Covering the resonator under water reduces $Q$ from 942 to 136 while increasing $R_{m}$ from $200 \Omega$ to $1.52 \mathrm{k} \Omega$. The resonant frequency shifts down by $0.55 \%$ relative to the nominal frequency in air of $5.41 \mathrm{MHz}$. Owing to the strong coupling provided by the SE mode, $R_{m}$ is only $1.52 \mathrm{k} \Omega$ despite a $Q$ of 136 in water. 


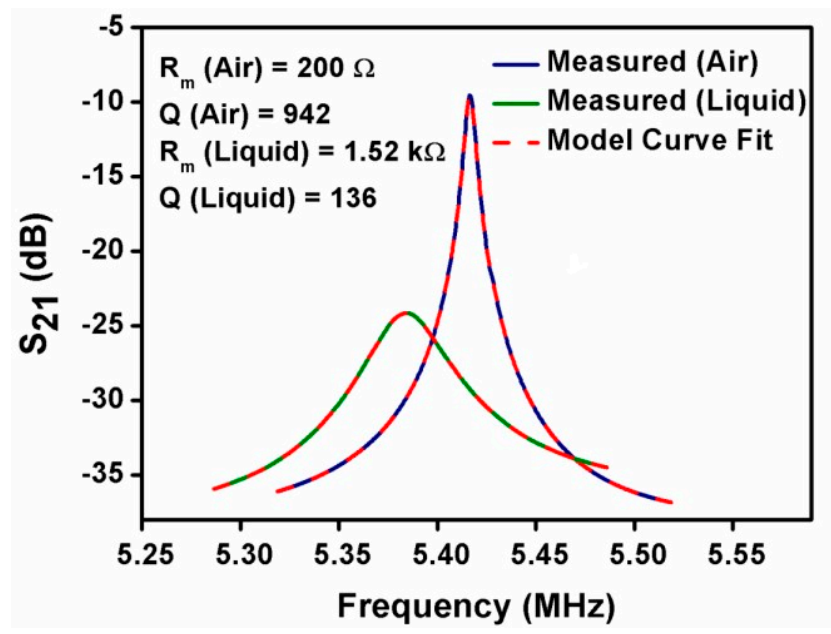

Figure 2. Measured electrical transmission of SE mode MEMS resonator in air (solid blue line) and liquid (solid green line) with model curve fit (dashed red line).

\subsection{Close-Loop Implementation}

Figure 3 depicts the circuit topology for sustaining the resonator in oscillation and narrowing the frequency linewidth. A transimpedance amplifier (TIA) converts the motional current (Iout) from the sense port of the resonator to an output voltage $\left(V_{\text {out }}\right)$ to deliver an open loop gain greater than 0 $\mathrm{dB}$ to satisfy the Barkhausen criterion. The transimpedance amplifier gain was adjusted according to the extracted value of $R_{m}$. $V_{\text {out }}$ is fed back to the drive port of the resonator to sustain oscillation. Figure 4a shows the output waveform of the oscillator measured on an oscilloscope with the sensor in air. Figure $4 \mathrm{~b}$ shows the corresponding frequency spectrum of the output measured using a Spectrum Analyzer (ROHDE \& SCHARZ) where we see that the oscillation peak is locked onto the resonant frequency of $5.41 \mathrm{MHz}$ with a $-3 \mathrm{~dB}$ bandwidth of $95 \mathrm{~Hz}$. Compared to measurement in open- loop, the sensor in closed-loop narrows down the frequency resolution from $3680 \mathrm{ppm}$ to $8.76 \mathrm{ppm}$. Work is currently underway to cancel the substantial feedthrough when the resonator is covered in water with the aim to provide sufficient phase shift to sustain oscillation with the resonator in water.

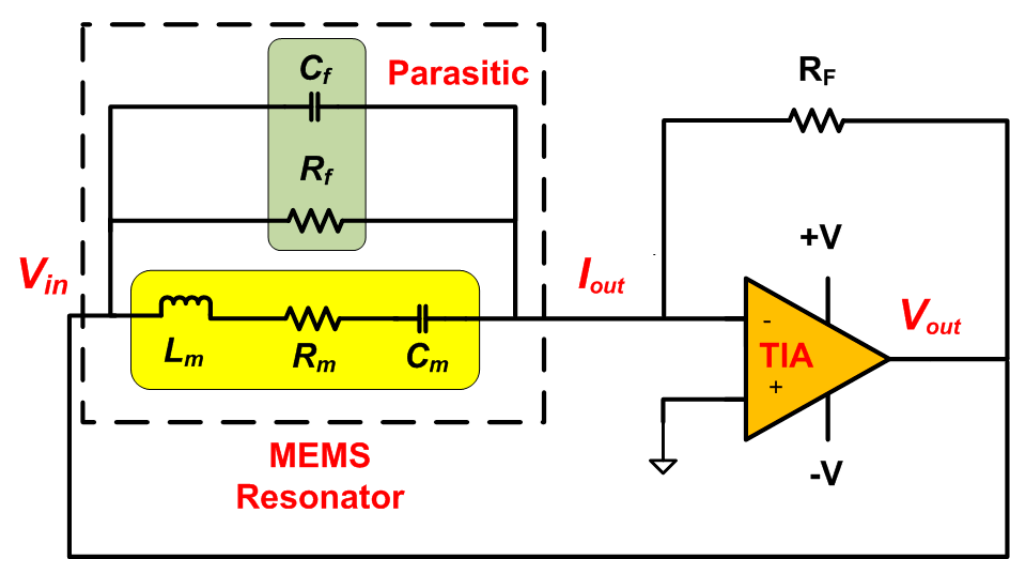

Figure 3. Circuit topology of self-sustained SE mode MEMS resonant mass sensor following a selfsustaining transimpedance amplifier. 


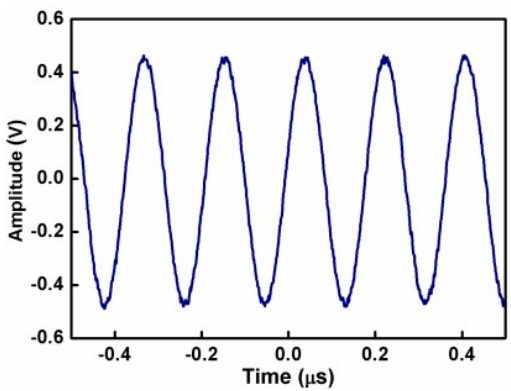

(a)

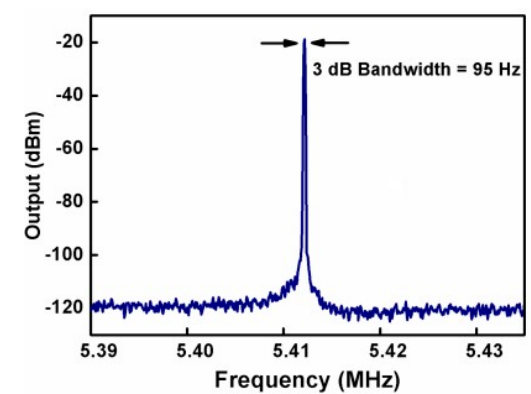

(b)

Figure 4. (a) Measured output waveform of SE mode resonant mass sensor in self-sustained oscillation; (b) Measured output frequency spectrum of SE mode resonant mass sensor in selfsustained oscillation.

\section{Conclusions}

In this paper, we have demonstrated a self-sustained square-extensional (SE) mode resonator oscillator for liquid phase mass sensing applications. As shown herein, $Q$ factors are greatly reduced when the device is covered in liquid compared to operating in air due to viscous damping in liquid. Incorporating a feedback loop to the MEMS resonator reduces the minimum resolvable frequency from $3680 \mathrm{ppm}$ to $8.76 \mathrm{ppm}$. Work is underway to demonstrate self-sustaining oscillation of the sensor in water.

Author Contributions: H.B. designed and performed the experiments and wrote the paper; A.A. designed the MEMS device; J.E.-Y.L. supervised the work and corrected the paper.

Acknowledgments: The work described in this paper was supported by grants from the Research Grants Council of Hong Kong under project numbers CityU 11206414 and 11211116.

Conflicts of Interest: The authors declare no conflict of interest.

\section{References}

1. Manzaneque, T.; Ruiz-Diez, V.; Hernando-Garcia, J.; Wistrela, E.; Kucera, M.; Schmid, U.; Sanchez-Rojas, J.L. Piezoelectric MEMS resonator-based oscillator for density and viscosity sensing. Sens. Actuators A 2014, 220, 305-315, doi:10.1016/j.sna.2014.10.002.

2. Zhao, X.; Pan, F.; Ashley, G.M.; Garcia-Gancedo, L.; Luo, J.; Flewitt, A.J.; Milne, W.I.; Lu, J.R. Label-free detection of human prostate-specific antigen (hPSA) using film bulk acoustic resonators (FBARs). Sens. Actuators B 2014, 190, 946-953, doi:10.1016/j.snb.2013.09.064.

3. Lin, A.T.-H.; Yan, J.; Seshia, A.A. Electrically addressed dual resonator sensing platform for biochemical detection. J. Microelectromech. Syst. 2012, 21, 34-43, doi:10.1109/JMEMS.2011.2174420.

4. Agache, V.; Blanco-Gomez, G.; Cochet, M.; Caillat, P. Suspended nanochannel in MEMS plate resonator for mass sensing in liquid. In Proceedings of the IEEE MEMS 2011, Cancun, Mexico, 23-27 January 2011.

5. Lee, J.; Shen, W.; Payer, K.; Burg, T.P.; Manalis, S.R. Toward attogram mass measurements in solution with suspended nanochannel resonators. Nano Lett. 2010, 10, 2537-2542, doi:10.1021/nl101107u.

6. Ziegler, D.; Klaassen, A.; Bahri, D.; Chmielewski, D.; Nievergelt, A.; Mugele, F.; Sader, J.E.; Ashby, P.D. Encased cantilevers for low-noise force and mass sensing in liquids. In Proceedings of the 27th IEEE MEMS 2014, San Francisco, CA, USA, 26-30 January 2014.

7. Kaajakari, V.; Mattila, T.; Oja, A.; Kiihamaki, J.; Seppa, H. Square-extensional mode single-crystal silicon micromechanical resonator for low phase noise oscillator applications. IEEE Electron. Device Lett. 2004, 25, 173-175, doi:10.1109/LED.2004.824840.

8. Lin, A.T.-H.; Yan, J.; Seshia, A.A. 2.2 MHz piezoresistive MEMS oscillator operating in air. In Proceedings of the Eurosensors XXIV, Linz, Austria, 5-8 September 2010. 